\title{
Protocol for a retrospective, controlled cohort study of the impact of a change in Nature journals' editorial policy for life sciences research on the completeness of reporting study design and execution
}

\author{
Fala Cramond ${ }^{1}$ Cadi Irvine ${ }^{1} \cdot$ Jing Liao $^{1} \cdot$ David Howells $^{2}$ • \\ Emily Sena $^{1} \cdot$ Gillian Currie $^{1} \cdot{\text { Malcolm } \text { Macleod }^{1}(D)}$
}

Received: 9 March 2016/Published online: 11 May 2016

(C) The Author(s) 2016. This article is published with open access at Springerlink.com

\begin{abstract}
In recent years there has been increasing concern about the rigor of laboratory research. Here we present the protocol for a study comparing the completeness of reporting of in vivo and in vitro research carried in Nature Publication Group journals before and after the introduction of a change in editorial policy (the introduction of a set of guidelines for reporting); and in similar research published in other journals in the same periods.
\end{abstract}

Keywords Risk of bias - Reporting · Methodological quality - Study design · Reporting guidelines

\section{Background}

Few publications describing in vivo research report taking measures which might reduce the risk of bias in their findings (Ioannidis et al. 2014; Macleod et al. 2015), and those which do not report such measures give inflated estimates of biological effects (Crossley et al. 2008; Hirst et al. 2014). Measures which might improve the quality of reports of in vivo research have been proposed (Kilkenny et al. 2010; Landis et al. 2012) and while these have been endorsed by a large number of journals there is evidence that this endorsement has not been matched by a substantial increase in the quality of published reports (Baker et al. 2014).

Poor replication of in vitro molecular and cellular biology studies has also been reported (Begley and Ellis 2012; Prinz et al. 2011) and linked in part to poor descriptions of the experimental and analytical details.

Electronic supplementary material The online version of this article (doi:10.1007/s11192-016-1964-8) contains supplementary material, which is available to authorized users.

\section{Malcolm Macleod}

Malcolm.Macleod@ed.ac.uk

1 Centre for Clinical Brain Sciences, University of Edinburgh, Edinburgh, Scotland, UK

2 School of Medicine, University of Tasmania, Hobart, TAS, Australia 
In May 2013 Nature Journals introduced a change in editorial policy which required authors of submissions in the life sciences to complete a checklist indicating whether or not they had taken certain measures which might reduce the risk of bias and to report key experimental and analytical details; and in their submission to detail where in the manuscript these issues were addressed (Anon 2013). The purpose of this study is to assess any impact of this change.

\section{Study design}

1.1. Aim To determine whether the implementation of a checklist for submissions has been associated with improved reporting of measures which might reduce the risk of bias.

1.2. Population Published articles accepted for publication in Nature journals, which describe research in the life sciences and which were submitted after May 1st 2013 and before November 1st 2014.

1.3. Intervention Mandatory completion of a checklist at the point of manuscript revision.

1.4. Comparator Published articles accepted for publication in Nature journals in the months preceding May 2013, which describe research in the life sciences.

1.5. Outcome Change in proportion of published studies which report measures which might reduce the risk of bias.

\section{Identification of relevant manuscripts}

\section{Nature publications}

One individual was specifically employed by Nature to select manuscripts based on a predefined criteria for inclusion. Following this, a Nature editorial administration manager reviewed selected manuscripts against the inclusion criteria and found that some manuscripts (fewer than $10 \%$ ) had been incorrectly included; they replaced these with manuscripts that they selected according to the inclusion algorithm. Across all participating Nature publications (see "Appendix 1") that describe primary research in the life sciences they identified papers accepted for publication with an initial submission date later than May 1st, 2013. Beginning with the current issue (volume corresponding to year 2015), they worked backwards in time, ensuring the submission date was after 1st May 2013, collecting papers until the number of studies required was reached ("Post intervention" group). They then used a similar process to identify papers submitted for publication before 1st May 2013, starting with the May 2013 issue and working backwards, ensuring that the date of submission was after 1st May 2011 ("pre-intervention" group). For each group, the intention was that 40 papers should be selected from Nature and 20 from each of the other titles. This would provide a total of 220 in each group and thus allow for any papers that did not fit the study to be excluded while ensuring the study includes 200 papers per group. The selection criteria, in addition to the dates of submission, were:

- the description of in vivo research (manuscripts that contain at least one non-human animal experiment, including rodents, flies, worms, zebrafish etc.) or in vitro research; 
- journal of publication of Nature, Nature Neuro; Nature Immunology; Nature Cell Biology, Nature Chemical Biology, Nature Biotechnology, Nature Methods, Nature Medicine, or Nature Structural and Molecular Biology;

- for the post intervention group, similar proportions for country of address for correspondence and journal.

Where no pre-intervention period match could be found with a submission date after 1st May 2011 (i.e. in the 2 years leading to the change in policy) then the non-matched post intervention publication was excluded from analysis and a replacement post intervention publication selected, as above, with a matching pre-intervention publication then identified, as described above. Publications describing research involving only human subjects were not to be included.

The published files corresponding to the publication pdfs (including the extended methods section, extended data and other supplementary materials) will be used to generate pdfs for analysis; these study pdfs will not include author names or affiliations, date, volume or page number; or any references (to allow a blinded assessment of outcome). These study pdfs will be presented for analysis in random order with a unique study identifier. This will be done by the Nature editorial administration manager; Nature editors and publishers will have no role in selecting the study manuscripts. All studies to be included will be listed in an MS Excel file. Each will then be allocated a random number between 0 and 1 using the RAND() command. They will then be sorted according to this number and a unique identifier corresponding to their position in this new sequence will be allocated. These studies, with this unique identifier, will be batch uploaded to a study computer [the Edinburgh Microsoft Access reporting quality scoring system (MARQSS)]. Information on group membership will be retained by the Nature editorial administration manager. Only once data analysis is complete will the coded group allocation (A and B) be revealed to the study team, along with coded research area, research area and country of origin. The study team will then prepare two reports, one to be used if group A is pre-change, and one to be used if group B is pre-change.

\section{Sister publications}

To investigate whether the changes apply only to Nature publications or whether there has been an increase in the general scientific literature of the proportion of published studies which report measures which might reduce the risk of bias, we will match all the Nature publications included with a sister publication (also selected from before and after May 2013 as described above) with the following methodology:

1. Using PubMed, enter the Nature publication title.

2. Add the "related citations for PubMed" result to the search builder.

3. In the second line search field "Date of publication" for related articles in the same calendar month (M0) and year (YYYY/MM).

4. Search.

5. In the results, start with the first result returned and establish that it was not published in an NPG Journal ("Appendix 1").

6. That being the case, then apply the study inclusion criteria (2.2.1), ensuring that there is a match on the in vivo/in vitro status between Nature publications and nonNature publications.

7. If the manuscript fulfils 5 and 6 select it for the study and retrieve the pdf. If not available from institutional subscriptions, seek the pdf of the paper and any supporting materials through an interlibrary loan or from the authors. 
8. Save a pdf file comprising the main manuscript and supporting information with a name in the form NPG $<\mathrm{xxx}>$ _pair_nonredact.

9. If the manuscript fails 5 or 6 repeat the search with the date of publication extended to 1 month earlier and 1 month later $(M-1$ to $M+1)$.

10. Repeat steps 5 through 9 until a matching publication is found.

11. Record the difference in calendar months relative to the date of publication of the index NPG article.

\section{Inclusion and exclusion criteria}

Manuscripts describing research in the life sciences, categorised as

1. those describing in vivo research (manuscripts that contain at least one non-human animal experiment, including rodents, flies, worms, zebrafish etc., and where either the exposure of interest, or the outcome, or both, are determined in whole living animals);

2. those describing exclusively vitro research (exclusively molecular and cellular biology); 3. those presenting both in vivo and in vitro experiments, as described above.

Publications describing research involving only human subjects alone will not be included-if there are animal studies included, the publication can still be used.

\section{Redaction}

To blind reviewers a redaction process will be carried out by a scientist in another institution:

1. Load the pdf to Adobe acrobat professional.

2. Identify date identifying information including publication date; volume and issue number; grant and funding information; and all references, including those which are in-line [i.e. (Smith et al. 2015)], those which are more integrated (i.e. "In a 2015 study by Smith and co-workers), and also in-line acknowledgements (i.e. "Reagent $\mathrm{x}$ kindly donated by R. Smith"). Additionally, any reference to years in the text was removed (i.e. "During the 1980 s...", "2015 census data...").

3. Use the redaction tool to redact these data.

4. Save the file with a name in the form $\mathrm{NPG}<\mathrm{xxx}>$ _pair_redact.

5. Upload this redacted file.

\section{Outcome assessment}

Manuscripts will be scored by two independent reviewers blinded both to intervention status (before or after the change in editorial policy) and to the scores from the other reviewer. While the source of the publication (NPG or not, which NPG journal) will not be redacted, this will likely be apparent to most reviewers anyway from the typeface, layout, house style etc. Discrepancies will be resolved by a third reviewer who will be blinded to the identity, and the scores of the previous reviewers.

We will recruit individuals experienced in the critical appraisal of published materials (through for instance involvement with previous systematic reviews). Reviewers will receive training using an online platform, supported by a training manual (supplementary material). They will be presented with manuscripts to score, and their assessment 
compared with a "gold standard" derived following review of a set of manuscripts each scored in house by the CAMARADES team. Once their concordance with that gold standard is greater than $80 \%$ for three successive manuscripts they will be considered to have been trained to a sufficient standard.

To score a manuscript they will log on to MARQSS, and will be allocated the next manuscript requiring to be scored for the first time. If all manuscripts held in MAQRSS at that time have already been scored once, then the reviewer will be allocated a manuscript for second screening. MAQRSS will ensure that reviewers do not receive for second review a manuscript for which they performed the first review. Once manuscripts have been scored twice MARQSS will compare those scores, item by item, and flag those manuscripts where there is a discrepancy. These discrepancies will then be resolved by a third, senior reviewer.

Where a manuscript describes both in vivo and in vitro research, data will be extracted for both. Where there is more than one experimental design under each of these headings, quality criteria must be reported for all experiments to be awarded the point, however some checklist items will have a 'partially' option.

Monitoring of outcome assessment after $10 \%$ of manuscripts have been scored and adjudicated; we will review performance and if there are questions that are highly represented in those resulting in disagreements we will review the training materials and amend them as appropriate.

\section{Primary outcome measure}

The proportion of publications in the intervention group describing in vivo research that meet the Landis criteria (item numbers \#2, \#3, \#4, \#5 of “Appendix 2"). For the purpose of this study, meeting the criteria means that for a study in which the parameter is relevant, it was reported as being performed or as not being performed. The evaluation principle is to determine if someone with reasonable domain-knowledge can understand the parameters of experimental design sufficiently to inform interpretation. These metrics will not be applied to exploratory studies, defined for this purpose as a study where hypothesis testing statistical analyses were not reported.

\section{Secondary outcome measures}

\section{In vivo research}

1. The change in prevalence of reporting of all of the Landis criteria (\#2,3,4 and 5 together).

2. For 3 other items identified in Table 1 and for the 4 individual components of the Landis checklist (\#1,2,3,4,5,7,9), the proportion of publications in the intervention and comparison groups considered to meet all of the relevant Nature checklist criteria.

3. For 2 other items identified in Table $1(\# 6,10)$, the change in the proportion of publications meeting that criteria between the comparison group and the intervention group.

\section{In vitro research}

1. The proportion of publications in the intervention group describing in vitro research that meet the Landis criteria (\#2, \#3, \#4, \#5 in the "Appendix 2"). 
2. For 3 other items identified in Table 1 and for the 4 individual components of the Landis checklist (\#1,2,3,4,5,9), the proportion of publications in the intervention and comparison groups considered to meet all of the relevant Nature checklist criteria.

3. For 2 other items identified in Table 1, the change in the proportion of publications meeting that criteria between the comparison group and the intervention group.

The papers that contain both in vitro and in vivo research will be scored for each type of research independently and will contribute to both of these secondary outcomes. For each secondary outcome the denominator for calculation of proportions will total number of papers reporting at least one in vivo or at least one in vitro experiment, respectively. We will also evaluate whether papers reporting both in vivo and in vitro results are more or less likely to be compliant on each secondary outcome measure.

\section{Tertiary outcome measures}

1. The proportion of publications in the intervention and comparison groups considered to meet all of the relevant Nature checklist criteria.

2. For each of the individual components of the Nature checklist, change in the proportion of publications meeting that criteria between the comparison group and the intervention group.

\section{Statistical considerations}

\section{Power calculations}

The operationalized Nature checklist comprises 77 items arranged under 19 headings. These items are very different in their form and, probably, in their relative importance. Not all will be relevant to all studies. Further, complete compliance with the Nature guidelines, while the desired outcome, is likely to remain uncommon; it would also be interesting to

Table 1 Detecting an editorially significant change or prevalence for specified items of the checklist

\begin{tabular}{|c|c|c|c|}
\hline \multicolumn{2}{|l|}{ Type of study ${ }^{a}$} & \multicolumn{2}{|c|}{$\begin{array}{l}\text { Editorially significant } \\
\text { change or prevalence }\end{array}$} \\
\hline Category of requirement & $\begin{array}{l}\text { Operationalized } \\
\text { checklist items }\end{array}$ & In vitro studies & Animal studies \\
\hline $\begin{array}{l}\text { Transparency in figures and } \\
\text { statistical description }\end{array}$ & $\# 1$ & $80 \%$ compliance & $80 \%$ compliance \\
\hline Data deposition & $\# 9$ & $80 \%$ compliance & $80 \%$ compliance \\
\hline Animals description & $\# 7$ & N/A & $80 \%$ compliance \\
\hline "Landis 4" & $\# 2,3,4,5$ & $15 \%$ increment & $80 \%$ compliance \\
\hline Reagents description (Ab and Cell line) & \#6 & $15 \%$ increment & $15 \%$ increment \\
\hline Code availability & \#10 & $15 \%$ increment & $15 \%$ increment \\
\hline
\end{tabular}

\footnotetext{
a The unit of assessment will be considered to be the publication; while editorial intervention could occur at any level, any impact of this would be mediated through an effect on the author, and this is therefore the smallest unit to which an intervention might be allocated at random. Where there is more than one experimental design under each of these headings, quality criteria must be reported for all experiments to be awarded the point, however some checklist items will have a 'partially' option. However, if there are two in vivo or in vitro experiments, one randomized non blinded one blinded non randomized, for the primary outcome we will consider the paper to be both randomized and blinded
} 
observe differences in the reporting of 27 key components (items under 1,2,3,4,5 and 7) of the checklist for animal studies, as well as relatively novel requirements (such as items under 6 and 10). The power calculations which follow are for in vivo research; for in vitro research they will be broadly similar.

\section{Primary outcome (see above)}

The Nature editorial team indicate that for the primary outcome they wish to know whether compliance with the "Landis 4", in aggregate, reaches $80 \%$. Table 2 shows the upper limits of observed compliance which might be found to be significantly lower than $80 \%$, at different levels of power, for different numbers of observations, using a one-sample proportion Wald test with $p<0.05$. 100 manuscripts would deliver $70 \%$ power, 150 manuscripts would deliver $80 \%$ power and 200 manuscripts would deliver $90 \%$ power to detect compliance $10 \%$ lower than this.

\section{Secondary outcome (in vivo research)}

Table 3 shows the changes in aggregate reporting of the Landis 4 criteria identified at different levels of power using a one sided two sample Chi squared test (STATA) (4 comparisons, $p<0.01$ ). Italic values are where an increase of $15 \%$ or less is detected.

\section{Secondary outcome (in vitro research)}

Table 4 shows the upper limit of observed compliance with the Landis 4 criteria which would be identified as being significantly less than $80 \%$ detected with different power using a one-sample proportion Wald test: (9 comparisons, $p<0.0051)$.

\section{Secondary outcome (see 3.2 (III))}

Table 5 shows the power to detect an absolute $15 \%$ increase in the reporting of each of \#6 and \#10, depending on the prevalence in the control group, using a one sided two sample Chi squared test (STATA), with 200 manuscripts in each group (9 comparisons (with 3.2.1.2, $p<0.0051)$. Italic values are where the power is $>0.80$.

\section{Statistical analysis plan}

We will use the Wald test to calculate the proportion (and $95 \%$ confidence intervals) of studies meeting the primary outcome, and for other outcomes where the proportion of manuscripts meeting individual components of the checklist is reported (3.2.1.2). For secondary outcomes, the $95 \%$ confidence boundaries will be adjusted to take account of the number of comparisons drawn (9 comparisons, $p<0.0051$, so $99.49 \%$ CIs). We will

Table 2 Upper limits of observed compliance at different power cut-offs

\begin{tabular}{llll}
\hline Sample size (per group) & 0.70 power $(\%)$ & 0.80 power $(\%)$ & 0.90 power $(\%)$ \\
\hline 100 & 70 & 68 & 66 \\
150 & 72 & 71 & 69 \\
200 & 73 & 72 & 70 \\
250 & 74 & 73 & 72 \\
\hline
\end{tabular}


Table 3 Changes in aggregate reporting of the Landis 4 criteria identified at different levels of power

\begin{tabular}{llll}
\hline $\begin{array}{l}\text { Prevalence } \\
\text { before }(\%)\end{array}$ & $\begin{array}{l}\text { Prevalence detected } \\
(0.70 \text { power })(\%)\end{array}$ & $\begin{array}{l}\text { Prevalence detected } \\
(0.80 \text { power })(\%)\end{array}$ & $\begin{array}{l}\text { Prevalence detected } \\
(0.90 \text { power })(\%)\end{array}$ \\
\hline 10 & 20 & 21 & 23 \\
20 & 32 & 34 & 36 \\
30 & 44 & 45 & 47 \\
40 & 54 & 56 & 58 \\
50 & 64 & 66 & 67 \\
\hline
\end{tabular}

Table 4 Upper limits of observed compliance at different power cut-offs

\begin{tabular}{llll}
\hline Sample size (per group) & 0.70 power $(\%)$ & 0.80 power $(\%)$ & 0.90 power $(\%)$ \\
\hline 100 & 65 & 64 & 61 \\
150 & 68 & 67 & 65 \\
200 & 70 & 69 & 67 \\
250 & 71 & 70 & 69 \\
\hline
\end{tabular}

Table 5 Power required to detect an absolute $15 \%$ increase in the reporting of items \#6 and \#10 of the Landis criteria items

\begin{tabular}{lll}
\hline Prevalence before $(\%)$ & Prevalence after $(\%)$ & Power \\
\hline 5 & 20 & 0.98 \\
10 & 25 & 0.92 \\
15 & 30 & 0.85 \\
20 & 35 & 0.79 \\
25 & 40 & 0.74 \\
30 & 45 & 0.70 \\
35 & 50 & 0.68 \\
40 & 55 & 0.67 \\
45 & 60 & 0.67 \\
50 & 65 & 0.68 \\
\hline
\end{tabular}

use Pearson Chi squared tests to test the significance of differences between groups for other secondary and tertiary outcomes, again with Holm-Boneferroni adjustment of critical $p$ values to account for multiple testing. We will also conduct an interrupted time series analysis using itsa in Stata, for the change in the proportion of publications meeting the primary outcome measure with dates of submission before or after May 2013 in the "treated" (NPG) group compared with the "control" (matched) group.

We will conduct sub-group analyses in groups defined by country of origin; categorisation of research; and whether the study is predominantly in silico; in vitro; in vivo; or involves human subjects.

\section{Ethical approval}

This study is a retrospective quality audit rather than a randomised trial. As such there are no ethical concerns. 


\section{Role of Nature in data analysis and data ownership}

The study dataset will belong to the investigators, and all decisions relating to data analysis and publication will be taken by the steering committee and will be independent of Nature. Once the manuscript is written, it will be shared with NPG and they will be invited to correct any errors of fact.

\section{Publication policy}

The entire set of derived data will be made available in a publically accessible, curated database within 12 months of completion of the study, or upon publication of the results of the study, whichever comes first. The study protocol will be lodged in a curated database. Because of the potential for conflict of interest the manuscript describing this work will not be submitted to any of the Nature journals but to a different journal.

Authorship of the main study report will include all those who have participated in the study design, planning, data collection or analysis.

\section{Committees}

The committee structure is defined to allow separation of interests.

\section{The study steering committee}

The study steering committee is responsible for strategic decisions regarding the study; and for data analysis, and for writing the first draft of the study report. It comprises Malcolm Macleod (University of Edinburgh, Chief Investigator and Chair), Emily Sena (University of Edinburgh), David Howells (School of Medicine, University of Tasmania).

\section{The study management committee}

The study management committee is responsible for ensuring the smooth running of the study, and at the outset comprised the study steering committee along with Veronique Kiermer (Nature). Dr Kiermer resigned from the study management committee in mid2015 when she left NPG, and was replaced by Dr. Sowmya Swaminathan.

Acknowledgments Funding The study is funded by a grant from the Laura and John Arnold Foundation.

\section{Compliance with ethical standards}

Conflict of interest None.

Open Access This article is distributed under the terms of the Creative Commons Attribution 4.0 International License (http://creativecommons.org/licenses/by/4.0/), which permits unrestricted use, distribution, and reproduction in any medium, provided you give appropriate credit to the original author(s) and the source, provide a link to the Creative Commons license, and indicate if changes were made.

\section{Appendix 1: List of Nature named journals}

- Nature 
- Nature Biotechnology

- Nature Cell Biology

- Nature Chemical Biology

- Nature Communications

- Nature Genetics

- Nature Immunology

- Nature Medicine

- Nature Methods

- Nature Neuroscience

- Nature Structural and Molecular Biology

\section{Appendix 2}

\section{See Table 6.}

Table 6 Operationalized checklist for Nature publication guidelines

\begin{tabular}{lll}
\hline Section title & $\begin{array}{l}\text { Item } \\
\text { number }\end{array}$ & Item \\
\hline
\end{tabular}

Figures and statistical representation of data (in vitro studies)

The following items should be found either in the figure legends or the methods section of the manuscript
1.1 The legend or the main text report the exact sample size (n) for each experimental group/condition, given as a number, not a range?

1.2 A description of the sample collection allowing the reader to understand whether the samples represent technical or biological replicates (including how many animals, litters, cultures, etc.)?

1.3 A statement of how many times the experiment shown was replicated in the laboratory?

1.4.1 Definitions of statistical methods and measures: If not a very common test (such as $t$ test, simple $\chi^{2}$ tests, Wilcoxon and Mann-Whitney tests, ANOVA), is the test described in the methods section?

1.4.2 Definitions of statistical methods and measures: Are tests reported as one-sided or two-sided?

1.4.3 Definitions of statistical methods and measures: Are there adjustments for multiple comparisons where this is appropriate?

1.4.4 Definitions of statistical methods and measures: Are the statistical test results (e.g., $p$ values, $F$ statistic etc.) presented?

1.4.5 Definitions of statistical methods and measures: Are the summary estimates defined as a median or average?

1.4.6 Definitions of statistical methods and measures: Are the error bars defined as s.d., s.e.m. or c.i.?

1.5.1 Implementation of statistical methods and measures: Do the authors show that their data meet the assumptions of the tests (e.g., normal distribution)?

1.5.2 Implementation of statistical methods and measures: Is there an estimate of variation within each group of data?

1.5.3 Implementation of statistical methods and measures: Is the variance similar (difference less than twofold) between the groups that are being statistically compared? 
Table 6 continued

\begin{tabular}{lll}
\hline Section title & $\begin{array}{l}\text { Item } \\
\text { number }\end{array}$ \\
\hline
\end{tabular}

For in vitro study: sample size

For in vitro study: exclusions 3

For in vitro study: randomization

For in vitro study: blinding

For in vitro study: reagents

For in vivo study: figures and statistical representation of data: for each figure (and each panel, where relevant), does the legend or the main text report
Does the manuscript describe how the sample size was chosen to ensure adequate power to detect a pre-specified effect size?

Does the manuscript include a statement about sample size estimate even if no statistical methods were used?

Does the manuscript describe if samples or animals were excluded from the analysis?

Are exclusion criteria defined?

Is it clear that the criteria were pre-established?

Does the manuscript describe which method of randomization was used to determine how samples/animals were allocated to experimental groups?

4.1 Does the manuscript describe include a statement about randomization even if no randomization was used?

Does the manuscript describe whether the investigator was blinded to the group allocation during the experiment and/or when assessing the outcome?

Does the manuscript include a statement about blinding even if no blinding was done?

Has every antibody used in this manuscript been profiled for use in the system under study, by either citation, catalog number, clone number or validation profile?

Was the source of cell lines provided?

Do the authors report whether the lines used have been authenticated recently (e.g., by STR profiling: within 1 year of use)?

Do the authors report whether the lines used have been tested for mycoplasma contamination recently (within 6 months of use)?

1.1 The legend or the main text report the exact sample size (n) for each experimental group/condition, given as a number, not a range?

1.2 A description of the sample collection allowing the reader to understand whether the samples represent technical or biological replicates (including how many animals, litters, cultures, etc.)?

1.3 A statement of how many times the experiment shown was replicated in the laboratory?

1.4.1 Definitions of statistical methods and measures: If not a very common test (such as $t$ test, simple $\chi^{2}$ tests, Wilcoxon and Mann-Whitney tests, ANOVA), is the test described in the methods section?

1.4.2 Definitions of statistical methods and measures: Where appropriate are tests reported as one-sided or two-sided?

1.4.3 Definitions of statistical methods and measures: Are there adjustments for multiple comparisons where this is appropriate?

1.4.4 Definitions of statistical methods and measures: Are the statistical test results (e.g., $p$ values, $F$ statistic etc.) presented? 
Table 6 continued

\begin{tabular}{lll}
\hline Section title $\begin{array}{ll}\text { Item } \\
\text { number }\end{array}$ & $\begin{array}{l}\text { Item } \\
1.4 .5\end{array}$ & $\begin{array}{l}\text { Definitions of statistical methods and measures: Are the } \\
\text { summary estimates defined as a median or average? } \\
\text { Definitions of statistical methods and measures: Are the error } \\
\text { bars defined as s.d., s.e.m. or c.i.? }\end{array}$ \\
$1.5 .1 \quad \begin{array}{l}\text { Implementation of statistical methods and measures: Do the } \\
\text { authors show that their data meet the assumptions of the tests } \\
\text { (e.g., normal distribution)? }\end{array}$ & $\begin{array}{l}\text { Implementation of statistical methods and measures: Is there an } \\
\text { estimate of variation within each experimental group of data } \\
\text { (e.g. Control vs Intervention group)? }\end{array}$ \\
$\begin{array}{c}\text { Implementation of statistical methods and measures: Is the } \\
\text { variance similar (difference less than twofold) between the } \\
\text { groups that are being statistically compared? }\end{array}$
\end{tabular}

For in vivo study: sample size 2

Does the manuscript describe how the sample size was chosen to ensure adequate power to detect a pre-specified effect size?

2.1 Does the manuscript include a statement about sample size estimate even if no statistical methods were used?

For in vivo study: exclusions

Does the manuscript describe if samples or animals were excluded from the analysis?

3.1 Are inclusion and exclusion criteria defined?

For in vivo study:

3.2 Is it clear that the criteria were pre-established?

randomization

Does the manuscript describe which method of randomization was used to determine how samples/animals were allocated to experimental groups?

4.1 Does the manuscript describe include a statement about randomization even if no randomization was used?

For in vivo study: blinding

Does the manuscript describe whether the investigator was blinded to the group allocation during the experiment and/or when assessing the outcome?

5.1 Does the manuscript include a statement about blinding even if no blinding was done?

For in vivo study: reagents

6.1 Has every antibody used in this manuscript been profiled for use in the system under study, by either citation, catalog number, clone number or validation profile?

For in vivo study: non-human 7.1.1 Does the study report the Species?

animal studies

7.1.2 Does the study report the Strain?

7.1.3 Does the study report the Sex?

7.1.4 Does the study report the age or weight (exact numbers required not ranges)?

7.2.1 Does the study include a statement of compliance with ethical regulations?

7.2.2 Does the study include the committee(s) approving the experiments?

For manuscript that include human studies

Does the manuscript identify the committee(s) approving the study protocol?

8.2 Does the manuscript include a statement confirming that informed consent was obtained from all subjects? 
Table 6 continued

\begin{tabular}{|c|c|c|}
\hline Section title & $\begin{array}{l}\text { Item } \\
\text { number }\end{array}$ & Item \\
\hline & 8.3 & $\begin{array}{l}\text { For publication of patient photos, does the study include a } \\
\text { statement confirming that consent to publish was obtained? }\end{array}$ \\
\hline & 8.4 & $\begin{array}{l}\text { Does the study report the clinical trial registration number (at } \\
\text { ClinicalTrials.gov or equivalent)? }\end{array}$ \\
\hline & 8.5 & $\begin{array}{l}\text { For phase II and III randomized controlled trials, does the } \\
\text { supplementary material include a CONSORT checklist? }\end{array}$ \\
\hline & 8.6 & $\begin{array}{l}\text { For tumor marker prognostic studies, does the supplementary } \\
\text { material include a REMARK checklist? }\end{array}$ \\
\hline \multirow[t]{10}{*}{ Data deposition } & 9.1 & $\begin{array}{l}\text { For studies describing Protein, DNA and RNA sequences, is an } \\
\text { accession code for deposited data in a public repository } \\
\text { provided? }\end{array}$ \\
\hline & 9.1 .1 & Does that accession code give access to the data? \\
\hline & 9.2 & $\begin{array}{l}\text { For studies describing Macromolecular structures, is an } \\
\text { accession code for deposited data in a public repository } \\
\text { provided? }\end{array}$ \\
\hline & 9.2 .1 & Does that accession code give access to the data? \\
\hline & 9.3 & $\begin{array}{l}\text { For studies describing Crystallographic data for small molecules, } \\
\text { is an accession code for deposited data in a public repository } \\
\text { provided? }\end{array}$ \\
\hline & 9.3 .1 & Does that accession code give access to the data? \\
\hline & 9.4 & $\begin{array}{l}\text { For studies describing microarray data, is an accession code for } \\
\text { deposited data in a public repository provided? }\end{array}$ \\
\hline & 9.4 .1 & Does that accession code give access to the data? \\
\hline & 9.5 & $\begin{array}{l}\text { For studies describing other data, is an accession code for } \\
\text { deposited data in a public repository provided? }\end{array}$ \\
\hline & 9.5 .1 & Does that accession code give access to the data? \\
\hline \multirow{5}{*}{$\begin{array}{l}\text { Computer code: for studies } \\
\text { using bespoke computer } \\
\text { based analysis }\end{array}$} & 10.1 & Is the computer source code provided with the paper? \\
\hline & 10.2 & Is the computer source code deposited in a public repository? \\
\hline & 10.2 .1 & Is that source code accessible? \\
\hline & 10.2 .2 & Does the source code function as described? \\
\hline & 10.3 & $\begin{array}{l}\text { If NO to } 10.1 \text { and } 10.2 \text {, does the manuscript indicate how the } \\
\text { computer source code can be obtained? }\end{array}$ \\
\hline Experiment type & 0.1 & $\begin{array}{l}\text { Does this publication describe in vivo experiments, in vitro/ } \\
\text { ex vivo experiments or both? }\end{array}$ \\
\hline Human related & 0.2 & Does this publication include human study? \\
\hline Computer code & 0.3 & Does this publication include computer code? \\
\hline Comments & 0.4 & Do you have other comments? If yes, please leave them here \\
\hline
\end{tabular}

\section{References}

Anon, J. (2013). Announcement: Reducing our irreproducibility. Nature, 496(7446), 398.

Baker, D., Lidster, K., Sottomayor, A., \& Amor, S. (2014). Two years later: Journals are not yet enforcing the ARRIVE guidelines on reporting standards for pre-clinical animal studies. PLoS Biology, 12, e1001756. 
Begley, C. G., \& Ellis, L. M. (2012). Drug development: Raise standards for preclinical cancer research. Nature, 483, 531-533.

Crossley, N. A., Sena, E., Goehler, J., Horn, J., van der Worp, B., Bath, P. M. W., et al. (2008). Empirical evidence of bias in the design of experimental stroke studies. A metaepidemiologic approach. Stroke, 39, 929-934.

Hirst, J. A., Howick, J., Aronson, J. K., Roberts, N., Perera, R., Koshiaris, C., \& Heneghan, C. (2014). The need for randomization in animal trials: An overview of systematic reviews. PLoS One, 9, e98856.

Ioannidis, J. P., Greenland, S., Hlatky, M. A., Khoury, M. J., Macleod, M. R., Moher, D., et al. (2014). Increasing value and reducing waste in research design, conduct, and analysis. Lancet, 383, 166-175.

Kilkenny, C., Browne, W. J., Cuthill, I. C., Emerson, M., \& Altman, D. G. (2010). Improving bioscience research reporting: The ARRIVE guidelines for reporting animal research. PLoS Biology, 8, e1000412.

Landis, S. C., Amara, S. G., Asadullah, K., Austin, C. P., Blumenstein, R., Bradley, E. W., et al. (2012). A call for transparent reporting to optimize the predictive value of preclinical research. Nature, 490, 187-191.

Macleod, M. R., Lawson, M. A., Kyriakopoulou, A., Serghiou, S., De, W. A., Sherratt, N., et al. (2015). Risk of bias in reports of in vivo research: A focus for improvement. PLoS Biology, 13, e1002273.

Prinz, F., Schlange, T., \& Asadullah, K. (2011). Believe it or not: How much can we rely on published data on potential drug targets? Nature Reviews Drug Discovery, 10, 712. 